\section{Dr. Dessein, et al reply}

\section{To the Editor:}

We thank Dr. Agilli and colleagues ${ }^{1}$ for their interest in our study of 217 patients with rheumatoid arthritis (RA) ${ }^{2}$. This investigation documented an independent relationship of leptin concentrations with carotid artery plaque in those with but not without a large cardiovascular disease (CVD) risk burden as represented by the presence of traditional risk factors ${ }^{2}$.

In our data analysis, we took into account the potential confounding or/and mediating effect of patient characteristics including a range of demographic features, lifestyle factors, anthropometric measures, RA characteristics, systemic inflammation, and kidney function. Interestingly, we found that the leptin concentration/atherosclerosis relationship was driven by the glomerular filtration rate and body mass index ${ }^{2}$. The included potential confounding or/and mediating variables in our analysis had been identified in a previous study that also showed an independent association of leptin concentrations with surrogate markers of early atherogenesis among young patients with RA in the present cohort $^{3}$.

Dr. Agilli and colleagues point out that, based on recently reported evidence, an additional 18 patient characteristics ${ }^{1}$ can influence circulating leptin levels, and therefore may have required consideration in our analysis. These include comorbid conditions such as major depression, chronic liver diseases, systemic lupus erythematosus (SLE), psoriasis, multiple sclerosis, and Helicobacter pylori infection, the use of glucocorticoids, and antipsychotic, antihypertensive, lipid-lowering, hormonal, insulin sensitivity-altering, and antidepressant medications, and dietary supplements such as vitamins A, D, and E, linoleic acid, and omega-3 fatty acids ${ }^{1}$.

Among our participants, none had concurrent chronic liver disease, SLE, psoriasis, or multiple sclerosis. H. pylori status was not assessed. None of the included patients used antipsychotic agents, and vitamins A and $\mathrm{E}$, linoleic acid, and omega-3 fatty acids were not prescribed by treating physicians.

Data on the remaining 8 potential confounding or/and mediating characteristics ${ }^{1}$ that could have been relevant in our analysis were recorded. These are given in Table 1. In this regard and as part of our routine practice, we systematically recorded the Arthritis Impact Measure Scales (AIMS) depression score ${ }^{4}$, because this is highly relevant in the present context $t^{4,5}$. Among patients with a large traditional CVD risk burden in the present study, the mean (SD) AIMS depression score was 3.0 (1.8), and $32.1 \%$ experienced a score of $>4$, which can indicate the

Table 1. Baseline characteristics and their associations with leptin concentrations in patients with rheumatoid arthritis who have a large traditional cardiovascular disease risk burden.

\begin{tabular}{|c|c|c|c|}
\hline \multirow[t]{2}{*}{ Characteristic } & & \multicolumn{2}{|c|}{$\begin{array}{c}\text { Association with Leptin } \\
\text { Concentrations* }\end{array}$} \\
\hline & & Partial R & $\mathrm{p}$ \\
\hline AIMS depression score $\geq 4$ & 32.1 & -0.284 & 0.05 \\
\hline Antidepressants & 32.1 & -0.178 & 0.2 \\
\hline Glucocorticoids & 1.9 & -0.147 & 0.3 \\
\hline Antihypertensives & 73.6 & -0.024 & 0.9 \\
\hline Lipid-lowering agents $^{\dagger}$ & 35.8 & 0.073 & 0.6 \\
\hline Insulin sensitivity-altering drugs & 28.3 & 0.332 & 0.02 \\
\hline Hormone replacement therapy ${ }^{\S}$ & 9.4 & 0.039 & 0.8 \\
\hline Vitamin D supplementation & 7.5 & 0.028 & 0.9 \\
\hline
\end{tabular}

*Associations were assessed in mixed regression models adjusted for age, sex, and race ${ }^{2,3} .{ }^{\dagger}$ Includes statin or/and ezetimibe. ${ }^{\ddagger}$ Comprises oral glucose lowering agents or/and insulin therapy. ${ }^{\S}$ Estrogen with or without progesterone used for menopausal symptoms. AIMS: Arthritis Impact Measure Scales. presence of clinical depression ${ }^{4}$. Apart from depression, the other potential confounding or/and mediating characteristics were the use of medications or groups of medications. This included vitamin D supplementation that, at the time of our study, formed part of treatment regimens for osteoporosis, which included a bisphosphonate or strontium ranelate. Notably, vitamin D is implicated in CVD risk in $\mathrm{RA}^{6,7}$.

As also shown in Table 1, among the 8 respective characteristics ${ }^{1}$, an AIMS depression score of $>4$ as well as the use of potential glucose sensitivity-altering medications were in fact associated with leptin concentrations in an analysis adjusted for demographic variables. This does support the issues raised by Dr. Agilli and colleagues ${ }^{1}$.

In our previously reported $s^{2} \mathrm{du}^{2}$, we found that a 1-SD increment in leptin concentration increased the OR for plaque 2.75 -fold (95\% CI 1.19 to $6.37 ; \mathrm{p}=0.01)$ after adjustment for potential confounding or/and mediating characteristics in a mixed regression model. Glucocorticoid therapy was used by only $1.9 \%$ of the participating patients with a large CVD risk burden, and the effect of glucocorticoid therapy on the leptin concentration/atherosclerosis relationship was not assessed, because none of the patients using the respective intervention had plaque. When we reevaluated the leptin concentration/carotid artery plaque relationship with the additional inclusion of any of the remaining 7 newly identified potential confounders or/and mediators including depression or any of the recorded interventions in the mixed regression model $^{2}$, the respective association persisted on each occasion (Table 2).

It is possible if not likely that some of our patients were using dietary supplements without our knowledge. Despite this, taken together, the comments by Dr. Agilli, et $a l^{1}$ and the results of the ensuing analysis as presented here strengthen our finding that leptin can contribute to atherogenesis, and consideration of circulating concentrations of this adipokine may be useful in CVD risk stratification among patients with $\mathrm{RA}^{2,3}$.

PATRICK H. DESSEIN, MC, FCP(SA), FRCP(UK), PhD; LINDA TSANG; ANGELA G. WOODIWISS, PhD; Cardiovascular Pathophysiology and Genomics Research Unit, School of Physiology, Faculty of Health Sciences, University of the Witwatersrand; AHMED SOLOMON, MBBCh, FCP(SA), Department of Rheumatology, Charlotte Maxeke Johannesburg Academic Hospital, Faculty of Health Sciences, University of the Witwatersrand, Johannesburg, South Africa. Supported by The South African Medical Research Council (grant MRC2008_DES) and the National Research Foundation. Address correspondence to Dr. P.H. Dessein, P.O. Box 1012, Melville 2109, Johannesburg, South Africa. E-mail: dessein@telkomsa.net

Table 2. Relationship of leptin concentrations (1 SD increment) with carotid artery plaque in patients with rheumatoid arthritis who have a large traditional cardiovascular disease risk burden.

\begin{tabular}{lcc}
$\begin{array}{l}\text { Adjusted Characteristic in Addition } \\
\text { to Previously Identified Confounders } \\
\text { or/and Mediators }\end{array}$ & OR $(95 \% \mathrm{CI})$ & $\mathrm{p}$ \\
\hline AIMS depression score $\geq 4$ & $2.51(1.05-5.96)$ & 0.03 \\
Antidepressants & $2.52(1.03-6.12)$ & 0.03 \\
Antihypertensives & $2.78(1.19-6.48)$ & 0.01 \\
Lipid-lowering agents $^{\dagger}$ & $2.76(1.19-6.41)$ & 0.01 \\
Glucose sensitivity-altering drugs & $6.01(1.70-21.28)$ & 0.004 \\
Hormone replacement therapy & $2.82(1.18-6.75)$ & 0.02 \\
Vitamin D supplementation & $2.98(1.25-7.10)$ & 0.01
\end{tabular}

*Includes Framingham score, race, C-reactive protein, body mass index, and glomerular filtration rate $^{2,3}$. Comprises statin or/and ezetimibe. †ncludes oral glucose-lowering agents or/and insulin therapy. ${ }^{\S}$ Estrogen with or without progesterone used for menopausal symptoms. AIMS: Arthritis Impact Measure Scales. 


\section{REFERENCES}

1. Agilli M, Aydin F, Gulcan Kurt Y, Cayci T. Evaluation of leptin with atherosclerosis in rheumatoid arthritis. J Rheumatol 2015;42:727.

2. Dessein PH, Tsang L, Woodiwiss AJ, Solomon A. Effect of traditional cardiovascular risk factors on the independent relationship of leptin with atherosclerosis in rheumatoid arthritis. J Rheumatol 2014;41:2087-9.

3. Dessein PH, Norton GR, Woodiwiss AJ, Tsang L, Solomon A. Age impacts on the independent relationships of leptin with cardiometabolic risk and surrogate markers of enhanced early atherogenesis in black and white patients with rheumatoid arthritis: a cross-sectional study. Rheumatol Int 2014;34:329-39.

4. Solomon A, Christian BF, Woodiwiss AJ, Norton GR, Dessein PH. Burden of depressive symptoms in South African healthcare patients with established rheumatoid arthritis: a case-control study. Clin Exp Rheumatol 2011;29:506-12.

5. Dessein P, Christian B, Woodiwiss AJ, Norton G, Solomon A. Public healthcare attendance associates with enhanced conventional and non-conventional atherosclerotic cardiovascular disease risk burdens in established rheumatoid arthritis. Clin Exp Rheumatol 2010;28:230-7.

6. Ranganathan P, Khalatbari S, Yalavarthi S, Marder W, Brook R, Kaplan MJ. Vitamin D deficiency, interleukin 17, and vascular function in rheumatoid arthritis. J Rheumatol 2013;40:1529-34.

7. Dessein PH. Vitamin D replacement therapy: a promising adjunct in cardiovascular risk management among patients with rheumatoid arthritis. J Rheumatol 2013;40:1463-5.

J Rheumatol 2015;42:4; doi:10.3899/jrheum.141536 